\title{
Correction: Testing the potential of a virtual reality neurorehabilitation system during performance of observation, imagery and imitation of motor actions recorded by wireless functional near-infrared spectroscopy (fNIRS)
}

\author{
Lisa Holper ${ }^{1,2^{*}}$, Thomas Muehlemann ${ }^{1,3}$, Felix Scholkmann ${ }^{1}$, Kynan Eng ${ }^{2}$, Daniel Kiper ${ }^{2}$ and Martin Wolf ${ }^{1}$
}

\section{Correction}

Following publication of our article [1], we realised that some of the statistical tests used were not appropriate. We have now conducted the appropriate statistical tests, and updated the relevant tables, figures five and six (Figures 1 and 2 here, respectively) and conclusions accordingly.

For both the unilateral and the bilateral groups, analyses were recalculated.

Intra-condition differences:

- original publication [1]: paired $t$-test using means per trial for unilateral and bilateral group

- update: one-way repeated measures ANOVA using means per subject for unilateral and bilateral group

Inter-condition differences:

- original publication [1]: one-way ANOVA using means per trial for unilateral and bilateral group
- update: one-way repeated measures ANOVA using means per subject for unilateral and bilateral group

\section{Conclusions}

For the unilateral group (Table 1 and Figure 1), no changes in significance levels were found in $\Delta\left[\mathrm{O}_{2} \mathrm{Hb}\right]$ signals. For the bilateral group (Table 2 and Figure 2), the main differences compared to the original publication are that the intra-condition differences $\left(\left[\mathrm{O}_{2} \mathrm{Hb}\right]_{\text {rest }}\right.$ versus $\left.\left[\mathrm{O}_{2} \mathrm{Hb}\right]_{\text {stim }}\right)$ for the two conditions 'Observation Right' $\left(\mathrm{O} \_\mathrm{R}, \mathrm{p}=0.077\right)$ and 'Observation Left' (O_L, $\mathrm{p}=0.080$ ) recorded over the ipsilateral hemisphere do not reach significant level any more. Hence, the paragraphs discussing the intra-condition significances in those two conditions (sections Observation, imagery and imitation and Bilateral oxygenation of the Discussion) are only applicable for the contralateral hemisphere. Further, in both groups changes in significance levels were found for $\Delta[\mathrm{HHb}]$. However, since the Discussion and Conclusion of the originally published article only focuses on the concentration changes found in $\Delta\left[\mathrm{O}_{2} \mathrm{Hb}\right]$, this aspect does not change these sections.

\footnotetext{
* Correspondence: holper@ini.phys.ethz.ch

${ }^{1}$ Biomedical Optics Research Laboratory (BORL), Division of Neonatology, Department of Obstetrics and Gynecology, University Hospital Zurich, Frauenklinikstrasse 10,8091, Zurich, Switzerland

${ }^{2}$ Institute of Neuroinformatics (INI), University of Zurich and ETH Zurich, Winterthurerstrasse 190, 8057, Zurich, Switzerland

Full list of author information is available at the end of the article
} 


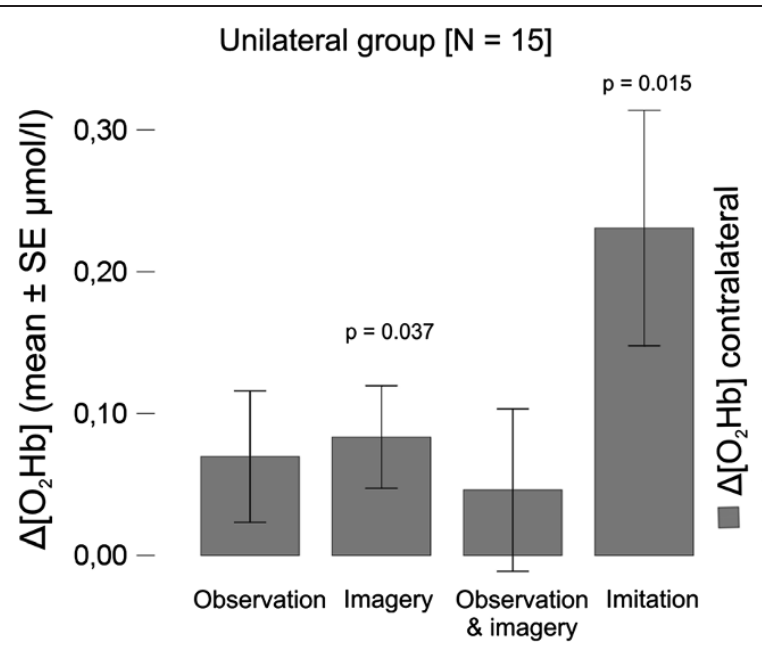

Figure 1 Unilateral group recorded over contralateral hemisphere: shown are the $\Delta\left[\mathrm{O}_{2} \mathrm{Hb}\right]$ amplitude changes with standard error of the mean (SEM) and statistical significances of repeated measures ANOVA.

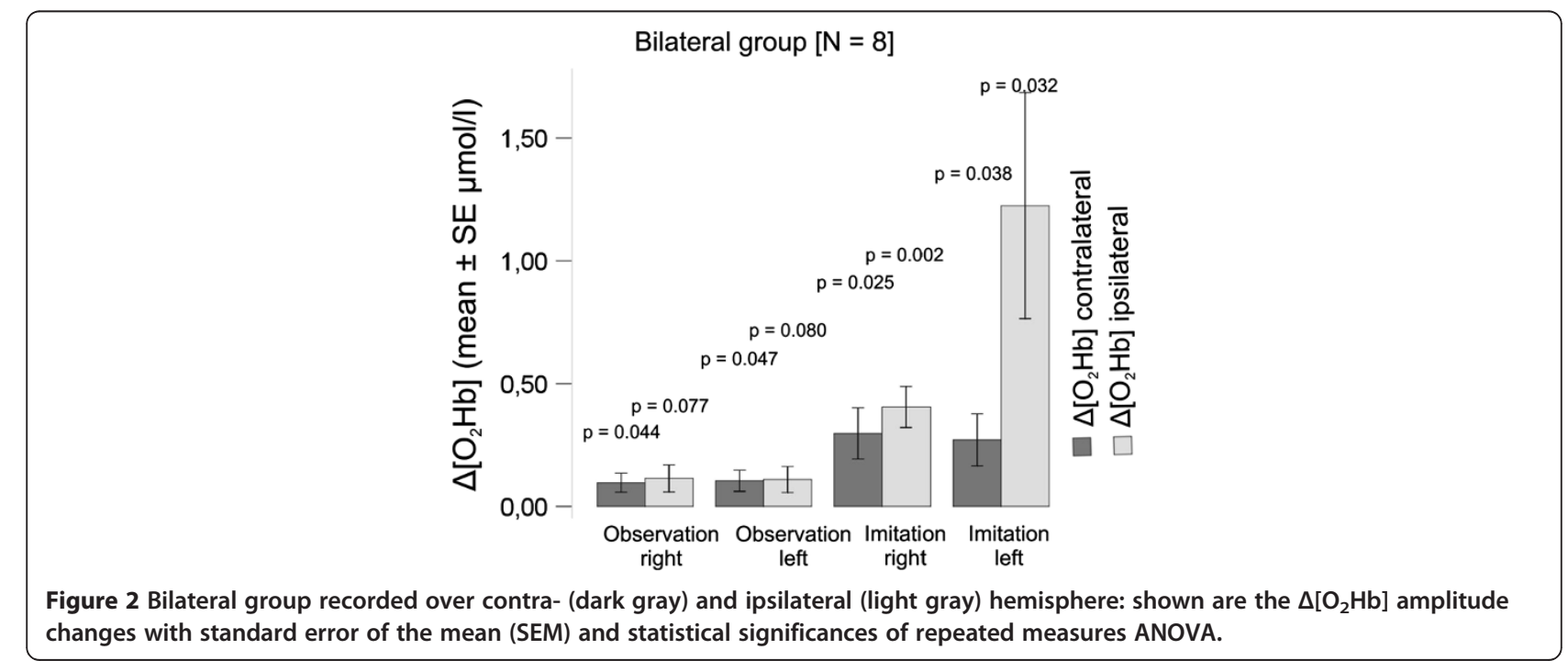


Table 1 Unilateral group

\begin{tabular}{|c|c|c|c|c|}
\hline Unilateral group [N = 15] & Observation & Motor imagery & Observation \& motor imagery & Imitation \\
\hline \multicolumn{5}{|c|}{ Left hemisphere (contralateral) $(\mu \mathrm{mol} / \mathrm{I} \pm \mathrm{SD})$} \\
\hline Mean $\Delta\left[\mathrm{O}_{2} \mathrm{Hb}\right]$ & $0.06953 \pm 0.1800$ & $0.0833 \pm 0.1404$ & $0.0460 \pm 0.2218$ & $0.2309 \pm 0.3212$ \\
\hline Mean $\triangle[\mathrm{HHb}]$ & $-0.0051 \pm 0.03855$ & $0.0356 \pm 0.0771$ & $-0.0089 \pm 0.0963$ & $0.0079 \pm 0.0832$ \\
\hline \multicolumn{5}{|c|}{ Intra-condition, ANOVA, repeated measures } \\
\hline$\left[\mathrm{O}_{2} \mathrm{Hb}\right]$ rest-stim & $p=0.157$ & $p=0.037^{*}$ & $p=0.435$ & $p=0.015^{*}$ \\
\hline [HHb] rest-stim & $p=0.612$ & $p=0.097$ & $p=0.727$ & $p=0.717$ \\
\hline \multicolumn{2}{|c|}{ Inter-condition ANOVA, repeated measures, post-hoc-tests, Bonferroni 0.05} & $\Delta[\mathrm{HHb}]$ & $\Delta\left[\mathrm{O}_{2} \mathrm{Hb}\right]$ & \\
\hline & $\mathrm{O}-\mathrm{Ml}$ & $p=0.347$ & $p=1.000$ & \\
\hline & $\mathrm{O}-\mathrm{O} \& \mathrm{Ml}$ & $p=1.000$ & $p=1.000$ & \\
\hline & $O-I M$ & $p=1.000$ & $p=0.286$ & \\
\hline & $\mathrm{Ml}-\mathrm{O} \& \mathrm{Ml}$ & $p=0.132$ & $p=1.000$ & \\
\hline & $\mathrm{MI}-\mathrm{IM}$ & $p=1.000$ & $p=0.622$ & \\
\hline & O\&MI - IM & $p=1.000$ & $p=0.321$ & \\
\hline Main effect on condition & & $p=0.253$ & $p=0.062$ & \\
\hline
\end{tabular}

(Top) Mean signal amplitudes ( $\mu \mathrm{mol} / \mathrm{I} \pm \mathrm{SD}$ ) of channels with significant concentration changes. Separate calculations for increases in $\left[\mathrm{O}_{2} \mathrm{Hb}\right]$, decreases in $[\mathrm{HHb}]$ in response to the four conditions for each group. Numbers were rounded to four decimal places. (Middle) Intra-condition statistical significance of the mean changes between $\left[\mathrm{O}_{2} \mathrm{Hb}\right]_{\text {rest }}$ and $\left[\mathrm{O}_{2} \mathrm{Hb}\right]_{\text {stim }}$ and $[\mathrm{HHb}]_{\text {rest }}$ and $[\mathrm{HHb}]_{\text {stim }}$ repeated measures ANOVA. (Bottom) Inter-condition statistical significance of mean changes of $\Delta\left[\mathrm{O}_{2} \mathrm{Hb}\right]$ and $\Delta[\mathrm{HHb}]$ between the four conditions using repeated measures ANOVA. Shown are post-hoc tests (with Bonferroni correction); significant values $(\mathrm{p} \leq 0.05)$ are highlighted by * (observation $=0$, motor imagery $=\mathrm{Ml}$, observation \& motor imagery $=0$ \& $\mathrm{Ml}$, imitation $=\mathrm{IM})$. 
Table 2 Bilateral group

\begin{tabular}{|c|c|c|c|c|}
\hline Bilateral group $[\mathrm{N}=8]$ & Observation right & Observation left & Imitation right & Imitation left \\
\hline \multicolumn{5}{|c|}{ Left hemisphere (contralateral) $(\mu \mathrm{mol} / \mathrm{l} \pm \mathrm{SD})$} \\
\hline Mean $\Delta\left[\mathrm{O}_{2} \mathrm{Hb}\right]$ & $0.1231 \pm 0.1506$ & $0.1231 \pm 0.1507$ & $0.3941 \pm 0.4598$ & $0.3715 \pm 0.4289$ \\
\hline Mean $\Delta[\mathrm{HHb}]$ & $-0.0056 \pm 0.0676$ & $-0.0408 \pm 0.0915$ & $0.0371 \pm 0.1131$ & $0.0474 \pm 0.0665$ \\
\hline \multicolumn{5}{|c|}{ Intra-condition, ANOVA, repeated measures } \\
\hline$\left[\mathrm{O}_{2} \mathrm{Hb}\right]$ rest-stim & $p=0.044^{*}$ & $p=0.047^{*}$ & $p=0.025^{*}$ & $p=0.038^{*}$ \\
\hline$[\mathrm{HHb}]$ rest-stim & $p=0.821$ & $p=0.247$ & $p=0.384$ & $p=0.084$ \\
\hline \multicolumn{2}{|c|}{ Inter-condition, ANOVA, repeated measures, post-hoc-tests, Bonferroni 0.05} & $\Delta[\mathrm{HHb}]$ & $\Delta\left[\mathrm{O}_{2} \mathrm{Hb}\right]$ & \\
\hline & O_R - O_L & $p=1.000$ & $p=1.000$ & \\
\hline & O_R - IM_R & $p=1.000$ & $p=0.519$ & \\
\hline & O_R - IM_L & $p=1.000$ & $p=0.862$ & \\
\hline & $\mathrm{OL}_{-}-\mathrm{IM} \_\mathrm{R}$ & $p=0.227$ & $p=0.486$ & \\
\hline & O_L - IM_L & $p=0.223$ & $p=0.777$ & \\
\hline & IM_R - IM_L & $p=1.000$ & $p=1.000$ & \\
\hline Main effect on condition & & $p=0.072$ & $p=0.119$ & \\
\hline \multicolumn{5}{|c|}{ Right hemisphere (ipsilateral) $(\mu \mathrm{mol} / \mathrm{I} \pm \mathrm{SD})$} \\
\hline Mean $\Delta\left[\mathrm{O}_{2} \mathrm{Hb}\right]$ & $0.1541 \pm 0.0735$ & $0.1957 \pm 0.1957$ & $0.4036 \pm 0.2097$ & $1.3728 \pm 1.6143$ \\
\hline Mean $\Delta[\mathrm{HHb}]$ & $-0.0113 \pm 0.0334$ & $0.0068 \pm 0.0274$ & $0.0235 \pm 0.0402$ & $0.7016 \pm 1.9167$ \\
\hline \multicolumn{5}{|c|}{ Intra-condition, ANOVA, repeated measures } \\
\hline$\left[\mathrm{O}_{2} \mathrm{Hb}\right]$ rest-stim & $p=0.077$ & $p=0.080$ & $p=0.002^{*}$ & $p=0.032^{*}$ \\
\hline [HHb] rest-stim & $p=0.367$ & $p=0.502$ & $p=0.142$ & $p=0.335$ \\
\hline \multicolumn{2}{|c|}{ Inter-condition, ANOVA, repeated measures, post-hoc-tests, Bonferroni 0.05} & $\Delta[\mathrm{HHb}]$ & $\Delta\left[\mathrm{O}_{2} \mathrm{Hb}\right]$ & \\
\hline & O_R - O_L & $p=1.000$ & $p=1.000$ & \\
\hline & O_R - IM_R & $p=0.445$ & $p=0.014^{*}$ & \\
\hline & O_R - IM_L & $p=1.000$ & $p=0.324$ & \\
\hline & $\mathrm{OL}_{-}-\mathrm{IM} \_\mathrm{R}$ & $p=1.000$ & $p=0.015^{*}$ & \\
\hline & O_L - IM_L & $p=1.000$ & $p=0.231$ & \\
\hline & IM_R - IM_L & $p=1.000$ & $p=0.710$ & \\
\hline Main effect on condition & & $p=0.384$ & $p=0.008^{*}$ & \\
\hline
\end{tabular}

(Top) Mean signal amplitudes ( $\mu \mathrm{mol} / \mathrm{I} \pm \mathrm{SD}$ ) of channels with significant concentration changes. Separate calculations for increases in $\left[\mathrm{O}_{2} \mathrm{Hb}\right]$, decreases in $[\mathrm{HHb}]$ in response to the four conditions for each group. Numbers were rounded to four decimal places. (Middle) Intra-condition statistical significance of the mean change between $\left[\mathrm{O}_{2} \mathrm{Hb}\right]_{\text {rest }}$ and $\left[\mathrm{O}_{2} \mathrm{Hb}\right]_{\text {stim }}$ and $[\mathrm{HHb}]_{\text {rest }}$ and $[\mathrm{HHb}]_{\text {stim }}$ using repeated measures ANOVA. (Bottom) Inter-condition statistical significance of mean changes of $\Delta\left[\mathrm{O}_{2} \mathrm{Hb}\right]$ and $\Delta[\mathrm{HHb}]$ between the four conditions using repeated measures ANOVA. Shown are post-hoc tests (with Bonferroni correction); significant values $(p \leq 0.05)$ are highlighted by * (observation left $=O \_L$, observation right $=O \_R$, imitation left $=I M \_L$, imitation right $\left.=I M \_R\right)$.

Received: 1 February 2013 Accepted: 1 February 2013

Published: 8 February 2013

\section{Reference}

1. Holper $L$, et al: Testing the potential of a virtual reality neurorehabilitation system during performance of observation, imagery and imitation of motor actions recorded by wireless functional nearinfrared spectroscopy (fNIRS). J Neuroeng Rehabil 2012, 7:57.

doi:10.1186/1743-0003-10-16

Cite this article as: Holper et al:: Correction: Testing the potential of a virtual reality neurorehabilitation system during performance of observation, imagery and imitation of motor actions recorded by wireless functional near-infrared spectroscopy (fNIRS). Journal of NeuroEngineering and Rehabilitation 2013 10:16.

\section{Submit your next manuscript to BioMed Central and take full advantage of:}

- Convenient online submission

- Thorough peer review

- No space constraints or color figure charges

- Immediate publication on acceptance

- Inclusion in PubMed, CAS, Scopus and Google Scholar

- Research which is freely available for redistribution 